\title{
The effect of miR-146a on STAT1 expression and apoptosis in acute lymphoblastic leukemia Jurkat cells
}

\author{
WEIHONG YAN ${ }^{1}$, HUA GUO $^{1}$, FENG SUO ${ }^{2}$, CHUNLING HAN $^{1}$, HUA ZHENG $^{1}$ and TONG CHEN ${ }^{1}$ \\ Departments of ${ }^{1}$ Pediatrics and ${ }^{2}$ Radiology, Dongying People's Hospital, Dongying, Shangdong 257091, P.R. China
}

Received April 18, 2016; Accepted November 2, 2016

DOI: $10.3892 / \mathrm{ol} .2016 .5395$

\begin{abstract}
The effect of miR-146a-dependent regulation of STAT1 on apoptosis in acute lymphoblastic leukemia (ALL) Jurkat cells was investigated. The miR-146a mimic and miR-146a inhibitor vectors were constructed in vitro, and experimental grouping was as follows: Control group (untreated Jurkat cells), empty vector group (Jurkat cells transfected with empty vector), agonist group (Jurkat cells transfected with miR-146a mimic) and the inhibitor group (Jurkat cells transfected with miR-146a inhibitor). Western blot analysis was used to observe the expression, respectively, of STAT1, p-STAT1 and Bcl-xL, and flow cytometry was used to test apoptosis in Jurkat cells. STAT1 and p-STAT1 expression in the agonist group was higher than that in the control and empty vector groups, but lower in the inhibitor group, and differences were statistically significant $(\mathrm{P}<0.05)$. The rate of apoptosis in the agonist group was significantly higher than that of the control group and blank vector group, and it was significantly lower in the inhibitor group $(\mathrm{P}<0.05)$. As a tumor suppressor, miR-146a can regulate expression of apoptosis-promoting factor STAT1, and anti-apoptosis factor $\mathrm{Bcl}-\mathrm{xL}$, and is able to promote apoptosis of ALL Jurkat cells.
\end{abstract}

\section{Introduction}

As a negative regulator of the acute-phase reaction in vivo, miR-146a plays an important role in various inflammatory and tumor related processes $(1,2)$. Deficiency of miR-146a can result in leukemia in mice (3). The study by Zhang et al (4) showed that miR-146a was the only miRNA species which was elevated in both childhood acute lymphoblastic leukemia (ALL) and acute myeloid leukemia (AML). The transcription factor, STAT1 exerts its pro-apoptotic function by inducing the expression of

Correspondence to: Dr Tong Chen, Department of Pediatrics, Dongying People's Hospital, 317 Nanyi Road, Dongying, Shangdong 257091, P.R. China

E-mail: chen_tong9@163.com

Key words: miR-146a, STAT1, acute lymphoblastic leukemia, Jurkat cells, apoptosis caspases or by inhibiting growth-promoting NF- $\mathrm{BB}$ signals (5). $\mathrm{Bcl}-\mathrm{xL}$ is a member of the anti-apoptotic Bcl-2 family protein, key regulators of the mitochondrial apoptosis pathway (6).

In the present study, we analyzed the effect of miR-146a on the apoptotic mechanism of ALL Jurkat cells, in order to provide a basis for clinical treatment.

\section{Materials and methods}

Cells. Jurkat cells were obtained from the Chinese Type Culture Collection Center of Wuhan University (Wuhan, China). They were cultured in RPMI-1640 supplemented with $10 \%$ fetal bovine serum (both from Gibco BRL, Gaithersburg, MD, USA) in an incubator set to $37^{\circ} \mathrm{C}$ and $5 \%$ $\mathrm{CO}_{2}$ (Forma 3110 model; Thermo Fisher Scientific, Waltham, MA, USA). Actively growing cells were selected for seeding in 12-well culture plates (Corning Inc., Corning, NY, USA) at a density of $1.5 \times 10^{6}$ cells/well, and then were returned to the incubator under the same growth conditions. Fresh Opti-MEM culture medium (Gibco BRL) was added to cells before transfection.

According to the manufacturer's instructions, Opti-MEM culture medium was used to dilute miR-146a mimic and miR-146a inhibitor (both from Guangzhou Ruibo Biotechnology Co., Ltd., Guangzhou, China) and Trans-EZ transfection reagent (Shanghai Shengbo Biotech Co., Ltd., Shanghai, China). Trans-EZ transfection reagent and miR-146a mimic were sufficiently mixed with the inhibitor to form stable transfection complexes.

Electrotransfection of miR-146a overexpression vector. Cell Line Nucleofector ${ }^{\circledR}$ Solutions V electrotransfection reagent was mixed with supplement according to the proportion of 4.5:1. Cells $\left(1 \times 10^{6}\right)$ were centrifuged and resuspended in $100-\mu 1$ electrotransfection reagent and allowed to incubate for $10 \mathrm{~min}$. Two micrograms of miR-146a overexpression vector (Zimmer, Shanghai, China) encoding DNA, $2 \mu \mathrm{g}$ negative control DNA vector, and $2 \mu \mathrm{g}$ pmaxGFP ${ }^{\circledR}$ vector were added to the cell suspensions and mixed. The product was then transferred to an electrotransfection cup, and the cover was fastened down. An Amaxa electrotransfection instrument (Bio-Rad, Berkeley, CA, USA) was used to perform the electrotransfection procedure, and the program was started. Transfection efficiency of the cells was observed under a fluorescence microscope (AX-70, Olympus, Tokyo, Japan) and images were captured. 
Experimental grouping. Jurkat cells were treated as follows for western blot analyses and FACS analysis of apoptosis: Control group (untreated Jurkat cells), empty vector group (Jurkat cell transfected with empty vector), agonist group (Jurkat cell transfected with miR-146a mimic) and inhibitor group (Jurkat cells transfected with miR-146a inhibitor).

Western blot analysis. Total protein was extracted and measured for purity according to a previous study (4). Protein samples were separated by SDS-PAGE electrophoresis (Bio-Rad mini vertical electrophoresis apparatus). Separated protein was then transferred to cellulose acetate membranes (NC membranes). Membranes were blocked in 5\% skim milk in TBST. Membranes were then incubated in primary antibody [mouse anti-STAT1, p-STAT1 and Bcl-xL mAbs, used at a dilution of 1:1,000 and rabbit polyclonal $\beta$-actin antibody were all obtained from BIOSS (Beijing, China) and used at a dilution of 1:500]. Secondary antibody (horseradish peroxidase-labeled secondary antibody was purchased from Sigma (St. Louis, MO, USA) and used at a dilution of 1:200. ECL reagent was used for signal development (Amersham Biosciences, Uppsala, Sweden). Membranes were exposed and photographed with a FluorChem 8000 gel-imaging analyzer (Alpha Innotech, San Leandro, CA, USA).

Flow cytometry (FCM) (FACS). We used an Annexin V/PI double-staining FCM reagent kit (C60 Biotech) and a flow cytometer (FACScalibur model; Becton-Dickinson, Franklin Lakes, NJ, USA) installed with CellQuest software to conduct the analysis.

Statistical analysis. SPSS 20.0 software (Chicago, IL, USA) was used to conduct statistical analyses. Data are expressed as mean \pm standard deviation, and single-factor ANOVA analysis was used for comparison among groups. $\mathrm{P}<0.05$ was considered to indicate a statistically significant difference.

\section{Results}

Expression of STAT1, p-STAT1 and Bcl-xL. STAT1 and p-STAT1 expression in the agonist (miR-146a mimic) group was higher than in the control group and empty vector group, and was lowest in the inhibitor group. The differences between groups were statistically significant $(\mathrm{P}<0.05)$. Bcl-xL expression in the agonist group was lower than that in the blank control group and blank vector group, while this expression
Table I. Expression of STAT1, p-STAT1 and Bcl-xL.

\begin{tabular}{lccc}
\hline Groups & STAT1 & p-STAT1 & Bcl-xL \\
\hline Blank control & $0.40 \pm 0.06$ & $0.33 \pm 0.05$ & $0.46 \pm 0.07$ \\
Blank vector & $0.41 \pm 0.05$ & $0.30 \pm 0.05$ & $0.43 \pm 0.06$ \\
Agonist & $0.47 \pm 0.09$ & $0.65 \pm 0.08$ & $0.20 \pm 0.03$ \\
Inhibitor & $0.35 \pm 0.06$ & $0.12 \pm 0.04$ & $0.55 \pm 0.05$ \\
F-value & 6.532 & 8.527 & 7.421 \\
P-value & 0.025 & 0.009 & 0.016 \\
\hline
\end{tabular}

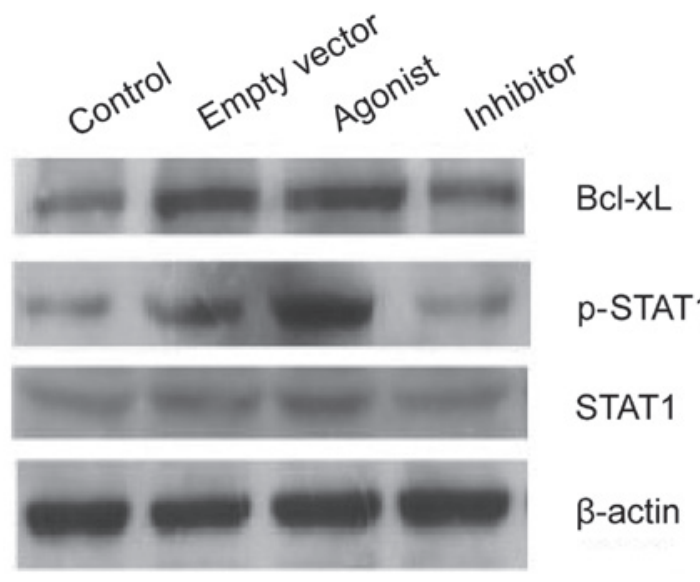

Figure 1. Western blot analysis. The levels of expression of STAT1, p-STAT1 and Bcl-xL in the different groups were assessed by western blot analysis. All experiments were repeated three times.

was the highest in inhibitor group. Differences were statistically significant $(\mathrm{P}<0.05)$ as shown in Table I and Fig. 1.

Jurkat cell apoptosis. Apoptosis in the agonist group was significantly higher than in the blank control group and blank vector group. Apoptosis was lowest in the inhibitor group agonist, $34.8 \pm 6.3 \%$ vs. control, $11.5 \pm 4.7 \%$ vs. empty vector, $12.3 \pm 4.6 \%$ vs. inhibitor, $4.9 \pm 1.5 \%$; $\mathrm{F}=18.629$, $\mathrm{P}<0.001)$ (Fig. 2).

\section{Discussion}

Expression of miR-146a is mainly located in hematopoietic stem cells and $\mathrm{T}$ lymphocytes (7). If miR-146a is overexpressed
Control

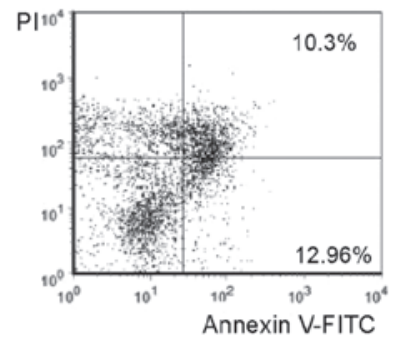

Empty vector

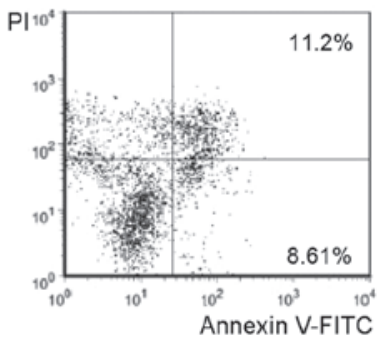

Agonist

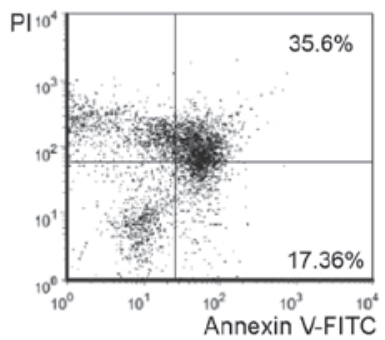

Inhibitor

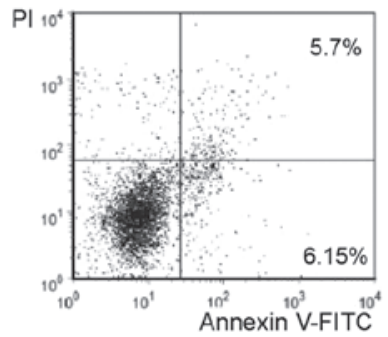

Figure 2. Detection of apoptosis. Apoptosis rates of the cells in each group as determined by Annexin-V/PI double-staining FCM. All experiments were repeated three times. FCM, flow cytometry. 
in hematopoietic stem cells and bone marrow transplantation is conducted, instantaneous marrow expansion will occur, erythropoiesis will be reduced and lymphocytopoiesis will be suppressed (8). Increased miR-146a expression is deleterious for marrow reconstruction in mice and reduces the survival rate of hematopoietic stem cells (9). Previous studies report that miR-146a regulates various aspects of the differentiation and survival of hematopoietic cells. Moreover, the effect of miR-146a expression in hematopoietic cells is similar to that of treatment with LPS (10). Expression of miR-146a was upregulated in the process of leukemia cell apoptosis induced by multiple drugs such as imatinib and all-trans retinoic acid, which may be related to the pro-apoptotic or anti-apoptotic activities of multiple transcription factors $(11,12)$.

The STAT family of proteins is responsible for transducing signals from growth factor receptors on the cytoplasmic membrane into the nucleus in order to regulate gene expression (12). Experiments using mutant cell lines and knockout mice have shown that STAT1 plays a significant role in interferon (IFN) $-\gamma$ and IFN- $\alpha / \beta$ mediated signaling. The activity of STAT1 is tightly regulated by multiple mechanisms including post-translational modification, regulatory proteins and modulation by other STAT family members (13-15). In a previous study, it was shown that (16) miR-146a can influence STAT1 activity via multiple pathways. Modifications such as phosphorylation, acetylation and ubiquitination can regulate the transcriptional activity of STAT1. Overexpression of miR-146a can induce cytoplasmic tyrosine kinase activity of JAK family proteins, promote phosphorylation of tyrosine-701 on STAT1, promote dimerization of STAT1 as well as increase its nuclear translocation and DNA binding activity (17). Downstrean of IFN signals, phosphorylation of serine residues is necessary for maximum expression of STAT1 target genes. The p38 MAPK kinase exerts an important influence on phosphorylation of serine on STAT1 serine site induced by miR-146a (18).

In mammals, there are two main pathways of apoptosis: The extrinsic pathway mediated by death receptors (DR) and the intrinsic pathway induced by mitochondria. Bcl-2 family proteins are equivalent to a molecular 'rheostat' which regulates the mitochondrial apoptosis pathway (19). Overexpression of Bcl-xL is regarded as being anti-apoptotic. Compared with Bcl-2, Bcl-xL can inhibit DISC formation and presentation and excitation of $\mathrm{Bid}$, which indicates that Bcl-xL can inhibit upstream signaling in TNF-induced apoptosis more effectively than Bcl-2 (20). Overexpression of Bcl-xL can inhibit disruption of the DeltaPsim (inner mitochondrial membrane potential) and reduce generation of ROS and MMP (21). The growth-promoting activity of Bcl-xL has been explored in a series of overexpression and gene knockout studies $(6,22)$.

From the present study, we can conclude that STAT1 and p-STAT1 expression was significantly increased when treated with miR-146a mimic over the control group and empty vector group. Additionally, STAT1 and p-STAT1 expression was lowest in cells treated with the miR-146a mimic inhibitor. Apoptosis in Jurkat cells was higher when treated with miR-146a mimic over the control group and empty vector group, and lowest in the inhibitor treated group. The differences were all statistically significant $(\mathrm{P}<0.05)$. This indicates that as a tumor suppressor, miR-146a can regulate the expression of the pro-apoptotic factor, STAT1 and the anti-apoptotic factor $\mathrm{Bcl}-\mathrm{xL}$ and is able to promote apoptosis of ALL Jurkat cells. However, future studies are required to confirm the findings.

\section{References}

1. Ye EA and Steinle JJ: miR-146a attenuates inflammatory pathways mediated by TLR4/NF- $\kappa \mathrm{B}$ and $\mathrm{TNF} \alpha$ to protect primary human retinal microvascular endothelial cells grown in high glucose. Mediators Inflamm 2016: 3958453, 2016.

2. Czajka AA, Wójcicka A, Kubiak A, Kotlarek M, Bakuła-Zalewska E, Koperski Ł, Wiechno W and Jażdżewski K: Family of microRNA-146 regulates RAR $\beta$ in papillary thyroid carcinoma. PLoS One 11: e0151968, 2016.

3. Lin Y, Zhang Q, Zhang HM, Liu W, Liu CJ, Li Q and Guo AY: Transcription factor and miRNA co-regulatory network reveals shared and specific regulators in the development of B cell and T cell. Sci Rep 5: 15215, 2015.

4. Zhang H, Luo XQ, Zhang P, Huang LB, Zheng YS, Wu J, Zhou H, Qu LH, Xu L and Chen YQ: MicroRNA patterns associated with clinical prognostic parameters and CNS relapse prediction in pediatric acute leukemia. PLoS One 4: e7826, 2009.

5. Adamaki M, Tsotra M, Vlahopoulos S, Zampogiannis A, Papavassiliou AG and Moschovi M: STAT transcript levels in childhood acute lymphoblastic leukemia: STAT1 and STAT3 transcript correlations. Leuk Res: Sep 11, 2015 (Epub ahead of print).

6. Lucas CM, Milani M, Butterworth M, Carmell N, Scott LJ, Clark RE, Cohen GM and Varadarajan S: High CIP2A levels correlate with an antiapoptotic phenotype that can be overcome by targeting BCL-XL in chronic myeloid leukemia. Leukemia: Feb 29, 2016 (Epub ahead of print).

7. Xu L, Zhong H, Wan H, Chen FY, Zhong J, Xiao F, Liu J and Shen L: miR-146a expression level as a novel putative prognostic marker for acute promyelocytic leukemia. Dis Markers 2014: $150604,2014$.

8. Duyu M, Durmaz B, Gunduz C, Vergin C, Yilmaz Karapinar D, Aksoylar S, Kavakli K, Cetingul N, Irken G, Yaman Y, et al: Prospective evaluation of whole genome microRNA expression profiling in childhood acute lymphoblastic leukemia. Biomed Res Int 2014: 967585, 2014.

9. Saki N, Abroun S, Soleimani M, Mortazavi Y, Kaviani S and Arefian E: The roles of miR-146a in the differentiation of Jurkat T-lymphoblasts. Hematology 19: 141-147, 2014.

10. Lionetti M, Musto P, Di Martino MT, Fabris S, Agnelli L, Todoerti K, Tuana G, Mosca L, Gallo Cantafio ME, Grieco V, et al: Biological and clinical relevance of miRNA expression signatures in primary plasma cell leukemia. Clin Cancer Res 19: 3130-3142, 2013.

11. Spinello I, Quaranta MT, Riccioni R, Riti V, Pasquini L, Boe A, Pelosi E, Vitale A, Foà R, Testa U, et al: MicroRNA-146a and AMD3100, two ways to control CXCR4 expression in acute myeloid leukemias. Blood Cancer J 1: e26, 2011.

12. Zhao C, Wang W, Yu W, Jou D, Wang Y, Ma H, Xiao H, Qin H, Zhang C, Lü J, et al: A novel small molecule STAT3 inhibitor, LY5, inhibits cell viability, colony formation, and migration of colon and liver cancer cells. Oncotarget 7: 12917-12926, 2016.

13. Midgley AC, Morris G, Phillips AO and Steadman R: $17 \beta$-estradiol ameliorates age-associated loss of fibroblast function by attenuating IFN- $\gamma / \mathrm{STAT} 1-d e p e n d e n t ~ m i R-7 ~ u p r e g u-$ lation. Aging Cell 15: 531-541, 2016.

14. Legrier ME, Bièche I, Gaston J, Beurdeley A, Yvonnet V, Déas O, Thuleau A, Château-Joubert S, Servely JL, Vacher S, et al: Activation of IFN/STAT1 signalling predicts response to chemotherapy in oestrogen receptor-negative breast cancer. Br J Cancer 114: 177-187, 2016.

15. Moles R, Bellon M and Nicot C: STAT1: A novel target of miR-150 and miR-223 is involved in the proliferation of HTLV-I-transformed and ATL cells. Neoplasia 17: 449-462, 2015.

16. Xu D, Han Q, Hou Z, Zhang C and Zhang J: miR-146a negatively regulates NK cell functions via STAT1 signaling. Cell Mol Immunol: Mar 21, 2016 (Epub ahead of print).

17. Wang S, Zhang X, Ju Y, Zhao B, Yan X, Hu J, Shi L, Yang L, Ma Z, Chen L, et al: MicroRNA-146a feedback suppresses T cell immune function by targeting Stat1 in patients with chronic hepatitis B. J Immunol 191: 293-301, 2013. 
18. Kutty RK, Nagineni CN, Samuel W, Vijayasarathy C, Jaworski C, Duncan T, Cameron JE, Flemington EK, Hooks JJ and Redmond TM: Differential regulation of microRNA-146a and microRNA-146b-5p in human retinal pigment epithelial cells by interleukin- $1 \beta$, tumor necrosis factor- $\alpha$, and interferon- $\gamma$. Mol Vis 19: 737-750, 2013.

19. Gény C, Rivière G, Bignon J, Birlirakis N, Guittet E, Awang K, Litaudon M, Roussi F and Dumontet V: Anacardic acids from Knema hookeriana as modulators of Bcl-xL/Bak and Mcl-1/Bid interactions. J Nat Prod 79: 838-844, 2016.

20. Sarosiek KA and Letai A: Directly targeting the mitochondrial pathway of apoptosis for cancer therapy with $\mathrm{BH} 3$ mimetics: recent successes, current challenges and future promise. FEBS J: Mar 21, 2016 (Epub ahead of print).
21. Matsumoto M, Nakajima W, Seike M, Gemma A and Tanaka N: Cisplatin-induced apoptosis in non-small-cell lung cancer cells is dependent on Bax- and Bak-induction pathway and synergistically activated by $\mathrm{BH} 3$-mimetic ABT-263 in p53 wild-type and mutant cells. Biochem Biophys Res Commun 473: 490-496, 2016.

22. Hennessy EJ: Selective inhibitors of Bcl-2 and Bcl-xL: balancing antitumor activity with on-target toxicity. Bioorg Med Chem Lett 26: 2105-2114, 2016. 